\title{
Optimal dynamic mean-variance asset-liability management under the Heston model
}

\author{
Jian Pan ${ }^{1 *}$, Zujin Zhang ${ }^{2}$ and Xiangying Zhou ${ }^{2}$
}

\section{"Correspondence:}

pan79610@163.com

${ }^{1}$ Key Laboratory of Jiangxi Province

for Numerical Simulation and

Emulation Techniques, Ganzhou,

China

Full list of author information is

available at the end of the article

\begin{abstract}
This paper studies a continuous-time mean-variance asset-liability management problem under the Heston model. Specifically, an asset-liability manager is allowed to invest in a risk-free asset and a risky asset whose price process is governed by the Heston model. By applying the Lagrange duality theorem and stochastic control theory, we derive the closed-form expressions of the efficient investment strategy and the efficient frontier. Moreover, we provide numerical experiments to analyze the sensitivity of the efficient frontier with respect to the relevant parameters in the Heston model.
\end{abstract}

Keywords: Continuous-time mean-variance; Asset-liability management; Heston model; Efficient investment strategy; Efficient frontier

\section{Introduction}

Asset-liability management (ALM) is essential for financial security systems such as banks, life insurance companies, property insurance companies and pension funds. In recent years, dynamic allocation strategies for mean-variance (M-V) ALM problems have been studied widely. These studies consider optimization problems of selecting optimal portfolios that can yield sufficient returns in compensating the corresponding liability. Sharpe and Tint [1] first consider an ALM problem under the static M-V framework. Keel and Müller [2] study a portfolio choice with liabilities and show that liabilities affect the efficient frontier. Based on the multi-period M-V framework, Leippold et al. [3] investigate a multi-period ALM problem and derive explicit expressions for the efficient investment strategy and the efficient frontier. By using the stochastic linear-quadratic control theory, Chiu and Li [4] study a continuous-time ALM problem where the risky assets' prices and the liability value are both governed by geometric Brownian motions. Xie et al. [5] also study a continuous-time ALM problem while the liability process is governed by a Brownian motion with drift. Chen et al. [6] and Chen and Yang [7] extend the work of Chiu and $\mathrm{Li}$ [4] and Leippold et al. [3] to the cases with a Markovian regime switching market. Chiu and Wong [8,9] apply the backward stochastic differential equation (BSDE) method to study the M-V ALM problems with cointegrated risky assets. Yao et al. [10, 11], respectively, consider a continuous-time M-V ALM problem and a multi-period M-V ALM problem with uncertain time horizon. Chiu and Wong [12] investigate a M-V ALM prob-

(c) The Author(s) 2018. This article is distributed under the terms of the Creative Commons Attribution 4.0 International License (http://creativecommons.org/licenses/by/4.0/), which permits unrestricted use, distribution, and reproduction in any medium, provided you give appropriate credit to the original author(s) and the source, provide a link to the Creative Commons license, and indicate if changes were made. 
lem with asset correlation risks, which are modeled by a multivariate Wishart process. Pan and Xiao [13] study an optimal M-V ALM problem with stochastic interest rates and inflation risks.

In most of the existing literature, it is standard to assume that the price of the risky asset (stock) follows a geometric Brownian motion, which implies that the volatility of risky asset price is a constant or a deterministic function. However, many phenomena, such as the volatility clustering, the volatility smile, the heavy-tailed nature of return distributions, etc., cannot be explained within the framework of deterministic volatility models. As natural extensions, stochastic volatility (SV) models have been proposed by many scholars such as the constant elasticity of variance (CEV) model (see Cox and Ross [14]), Stein-Stein model (see Stein and Stein [15]) and Heston model (see Heston [16]), where the CEV model and the Heston model have widely used in portfolio selection problems. Zhang and Chen [17] investigate a M-V ALM problem under the CEV model and derive the corresponding efficient investment strategy and efficient frontier by using the method of BSDE. Li et al. [18] consider a derivative-based optimal investment strategy for a M-V ALM problem under the Heston model and apply the BSDE method to obtain the explicit expressions of the efficient investment strategies and efficient frontiers for three special cases. In this paper, we also consider a continuous-time M-V ALM problem under the Heston model. Different from the work of Li et al. [18], we use a generalized Brownian motion to characterize the liability process, in which the expected growth rate of liabilities is cointegrated with the volatility of risky assets. Moreover, we apply the HamiltonJacobi-Bellman (HJB) equation method to derive the closed-form expressions of the efficient investment strategy and the efficient frontier. To the best of our knowledge, this problem has not yet received a complete treatment in the existing literature. The reason is that when both the Heston model and the more general liability model are introduced, this ALM problem becomes more complicated for adding the two new state variables. The dimension enlargement in the state space drastically increases the difficulty level in solving the associated HJB equation which comes from the dynamic programming approach. Here we need to point out that to reduce the dimension and obtain the closed-form expression, many scholars directly absorb the liability process into the dynamic process of assets instead of the traditional surplus process (the difference of liability from the asset). To solve this problem, we first apply the Lagrange multiplier method to transform the original problem into a standard stochastic optimal control problem and establish the corresponding extended $\mathrm{HJB}$ equation. Then we obtain the closed-form expression of the optimal investment strategy by solving the extended HJB equation. Furthermore, by the Lagrange duality theorem, we derive the efficient investment strategy and efficient frontier of this M-V ALM problem explicitly, which is our main contribution. We also discuss the effects on the efficient frontier of the stochastic volatility model parameters.

The remainder of this paper is organized as follows. Section 2 formulates the dynamic M-V ALM problem under the Heston model. Section 3 gives the efficient investment strategy and efficient frontier of the M-V ALM problem by applying the stochastic control theory and partial differential equation (PDE) method. Section 4 provides numerical examples to analyze the sensitivity of the efficient frontier based on the Heston model. Section 5 concludes this paper. 


\section{Problem formulation}

In this section, we introduce the financial market and establish the optimal dynamic $\mathrm{M}-\mathrm{V}$ ALM problem under the Heston model.

\subsection{The financial market}

Let $\left(\Omega, F,\left\{F_{s}\right\}_{t \leq s \leq T}, \mathrm{P}\right)$ be a filtered complete probability space satisfying the usual conditions, where $T>0$ is a finite constant representing the investment time horizon; $\left\{F_{s}\right\}_{t \leq s \leq T}$ is generated by two standard one-dimensional Brownian motions $W_{S}(t)$ and $W_{m}(t), \mathrm{P}$ is a real-world probability measure, and the expectation with respect to $\mathrm{P}$ is denoted $\mathrm{E}[\cdot]$.

In this probability space, we consider the manager who can invest in a risk-free asset (cash) and a risky asset (stock). The price process of the risk-free asset $B(t)$ is governed by

$$
\frac{d B(t)}{B(t)}=r d t, \quad B(0)=B_{0}>0
$$

where the constant $r$ represents the risk-free interest rate. The price process of the risky asset $S(t)$ follows the Heston model,

$$
\begin{cases}\frac{d S(t)}{S(t)}=\left[r+\lambda_{1} m(t)\right] d t+\sqrt{m(t)} d W_{S}(t), & S(0)=S_{0}>0, \\ d m(t)=k[\theta-m(t)] d t+\sigma_{m} \sqrt{m(t)} d W_{m}(t), & m(0)=m_{0}>0\end{cases}
$$

where $\lambda_{1}>0$ is a constant coefficient capturing the market price of the risk factor $W_{S}(t)$; $k, \theta$ and $\sigma_{m}$ are all positive constants and denote the mean-reversion rate, the long-run mean and the volatility coefficient of the instantaneous variance process $m(t)$, respectively; $W_{S}(t)$ and $W_{m}(t)$ are correlated with a constant correlation coefficient $\rho_{S m} \in[-1,1]$, namely, $\operatorname{Cov}\left(d W_{S}(t), d W_{m}(t)\right)=\rho_{S m} d t$.

Assumption 1 To ensure that $m(t)>0$, there exist parameters $k, \theta$ and $\sigma_{m}$ such that $2 k \theta \geq \sigma_{m}^{2}$.

In what follows, we consider the situation where the manager endowed with an initial wealth $X(0)=X_{0}>0$ at time 0 plans to invest in the financial market dynamically in the horizon $[0, T]$. Let $\pi(t)$ be the proportion of money invested in the stock at time $t$, and suppose that there are no transaction costs as well as other restrictions in the market. According to Eqs. (1) and (2), the dynamic of the total asset process $X(t)$ is

$$
\begin{aligned}
\frac{d X(t)}{X(t)} & =\pi(t) \frac{d S(t)}{S(t)}+[1-\pi(t)] \frac{d B(t)}{B(t)} \\
& =\left[r+\lambda_{1} \pi(t) m(t)\right] d t+\pi(t) \sqrt{m(t)} d W_{S}(t) .
\end{aligned}
$$

Here $\pi(t)$ is called an admissible portfolio strategy, i.e., if $\pi(t)$ is $F$-adapted, $\mathrm{E}\left[\int_{0}^{T} \pi^{2}(s) d s\right]<$ $+\infty$ and the stochastic differential equation in Eq. (3) together with $X_{0}$ has a unique strong solution $X(\cdot)$ corresponding to $\pi(\cdot)$. The set of all admissible portfolio strategies is denoted by $\Pi$.

On the other hand, in the course of investment practice, the investor may encounter uncontrollable liabilities and assume that the accumulative liability process $L(t)$ is described 
by

$$
d L(t)=\left[\alpha(t)+\lambda_{2} m(t)\right] d t+\sigma_{L} \sqrt{m(t)} d W_{S}(t), \quad L(0)=L_{0}>0,
$$

where $\sigma_{L} \sqrt{m(t)}$ is the volatility of $L(t)$ and $\sigma_{L}$ is a non-negative constant. Moreover, $\alpha(t)+$ $\lambda_{2} m(t)$ denotes the appreciation rate of $L(t)$, where $\alpha(t)$ is assumed to be a deterministic function of time $t$ and $\lambda_{2}$ is a constant.

Remark 1 To derive an explicit solution, we assume that the random term of liabilities is $\sqrt{m(t)} d W_{S}(t)$ in the interval $(t, t+d t)$.

\subsection{The mean-variance asset-liability management optimization problem}

In ALM models, one main concern is the surplus which is the difference of asset value and liability value. Thus ALM is also known as the surplus management. Let $Y(t)=X(t)-L(t)$ be the surplus. Then, by Eqs. (3) and (4), $Y(t)$ satisfies the following stochastic differential equation:

$$
\begin{aligned}
d Y(t)= & {\left[(Y(t)+L(t))\left(r+\lambda_{1} \pi(t) m(t)\right)-\alpha(t)-\lambda_{2} m(t)\right] d t } \\
& +\left[(Y(t)+L(t)) \pi(t)-\sigma_{L}\right] \sqrt{m(t)} d W_{S}(t),
\end{aligned}
$$

where the initial value $Y(0)=X_{0}-L_{0}>0$.

With the initial surplus $Y(0)$, the manager's objective is to find a strategy $\pi(t) \in \Pi$ to minimize the variance of the terminal surplus for a given level of the expected terminal surplus. More specifically, we consider the following optimization problem:

$$
\left\{\begin{array}{l}
\min _{\pi(t) \in \Pi} \operatorname{Var}[Y(T)]=\mathrm{E}[Y(T)-C]^{2}, \\
\text { subject to }\left\{\begin{array}{l}
\mathrm{E}[Y(T)]=C, \\
Y(t) \text { satisfies Eq. (5). }
\end{array}\right.
\end{array}\right.
$$

The solution $\pi^{*}(t)$ to Problem (6) is called an efficient investment strategy for $C \geq C^{*}$, where $C^{*}$ is the expected terminal surplus corresponding to the global minimum variance of the terminal surplus over all feasible strategies. The point $(\operatorname{Var}[Y(T)], \mathrm{E}[Y(T)])$ corresponds to an efficient investment strategy is called an efficient point. The set of all efficient points forms the efficient frontier in the variance-mean plane.

\section{Solution of the optimization problem}

In this section, we apply the Lagrange multiplier technique and stochastic control method to obtain a closed-form solution of Problem (6).

\subsection{Transformation of the original problem}

As widely adopted in the literature, we apply the Lagrange multiplier technique to deal with the constraint $\mathrm{E}[Y(T)]=C$. Define

$$
\begin{aligned}
J(Y(t), t ; \pi(t), \gamma) & =\mathrm{E}[Y(T)-C]^{2}+2 \gamma \mathrm{E}[Y(T)-C] \\
& =\mathrm{E}[Y(T)+\gamma-C]^{2}-\gamma^{2},
\end{aligned}
$$


where $\gamma \in \mathrm{R}$ is the Lagrange multiplier. Then by the Lagrange duality theorem (see Luenberger [19]), the original $M-V$ portfolio selection problem (6) is equivalent to the following max-min stochastic control problem:

$$
\left\{\begin{array}{l}
\max _{\gamma \in \mathrm{R}} \min _{\pi(t) \in \Pi} J(Y(t), t ; \pi(t), \gamma)=\mathrm{E}[Y(T)+\gamma-C]^{2}-\gamma^{2} \\
\text { subject to } Y(t) \text { satisfies Eq. (5). }
\end{array}\right.
$$

Clearly, to solve the above max-min stochastic control problem (8), we first need to consider the following quadratic loss minimization problem:

$$
\left\{\begin{array}{l}
\min _{\pi(t) \in \Pi} J_{0}(Y(t), t ; \pi(t), \gamma)=\mathrm{E}[Y(T)-u]^{2}, \\
\text { subject to } Y(t) \text { satisfies Eq. (5), }
\end{array}\right.
$$

where $u=C-\gamma$.

Problem (9) can be solved by using the stochastic control method. We now consider a truncated form of Problem (9) beginning at time $t$ and define the corresponding value function as

$$
H(t, m, y, l)=\inf _{\pi(t) \in \Pi} \mathrm{E}\left\{[Y(T)-u]^{2} \mid m(t)=m, Y(t)=y, L(t)=l\right\}
$$

with the boundary condition $H(T, m, y, l)=(y-u)^{2}$.

If $H(t, m, y, l) \in \mathrm{C}^{1,2,2,2}\left([0, T] \times \mathrm{R}^{+} \times \mathrm{R} \times \mathrm{R}\right)$, then by the principle of dynamic programming, $H(t, m, y, l)$ satisfies the following $\mathrm{HJB}$ equation:

$$
\begin{aligned}
H_{t} & +k(\theta-m) H_{m}+\frac{\sigma_{m}^{2}}{2} m H_{m m}+\left[\alpha(t)+\lambda_{2} m\right] H_{l}+\frac{\sigma_{L}^{2}}{2} m H_{l l} \\
& +\rho_{S m} \sigma_{m} \sigma_{L} m H_{m l}+\left[(y+l) r-\alpha(t)-\lambda_{2} m\right] H_{y}-m \sigma_{L}\left[\sigma_{L} H_{y l}\right. \\
& \left.+\sigma_{m} \rho_{S m} H_{y m}\right]+\inf _{\pi(t) \in \Pi}\left\{(y+l) \lambda_{1} \pi(t) m H_{y}+(y+l) m \pi(t)\left[\sigma_{L} H_{y l}\right.\right. \\
& \left.\left.+\sigma_{m} \rho_{S m} H_{y m}\right]+\frac{\left[(y+l) \pi(t)-\sigma_{L}\right]^{2} m}{2} H_{y y}\right\}=0,
\end{aligned}
$$

where $H$ is short for $H(t, m, y, l), H_{t}, H_{m}, H_{y}, H_{l}, H_{m m}, H_{y y}, H_{y l}, H_{l m}, H_{y m}$ and $H_{l l}$ denote the partial derivatives of first and second orders with respect to $t, m, y$ and $l$, respectively.

We first assume that $H_{y y}>0$, which will be verified later. The first-order condition for the optimization problem in the HJB Eq. (11) yields the optimal control as

$$
\pi^{*}(t)=\frac{\sigma_{L} H_{y y}-\sigma_{L} H_{y l}-\sigma_{m} \rho_{S m} H_{y m}-\lambda_{1} H_{y}}{(y+l) H_{y y}} .
$$

Substituting (12) into (11), after simplification, we have

$$
\begin{gathered}
H_{t}+k(\theta-m) H_{m}+\frac{\sigma_{m}^{2}}{2} m H_{m m}+\left[\alpha(t)+\lambda_{2} m\right] H_{l}+\frac{\sigma_{L}^{2}}{2} m H_{l l} \\
+\rho_{S m} \sigma_{m} \sigma_{L} m H_{m l}+\left[(y+l) r-\alpha(t)+m \lambda_{1} \sigma_{L}-\lambda_{2} m\right] H_{y}
\end{gathered}
$$




$$
\begin{aligned}
& -\frac{m}{2 H_{y y}}\left[\sigma_{L}^{2} H_{y l}^{2}+\lambda_{1}^{2} H_{y}^{2}+\sigma_{m}^{2} \rho_{S m}^{2} H_{y m}^{2}+2 \lambda_{1} \sigma_{L} H_{y} H_{y l}\right. \\
& \left.+2 \sigma_{m} \sigma_{L} \rho_{S m} H_{y l} H_{y m}+2 \lambda_{1} \sigma_{m} \rho_{S m} H_{y} H_{y m}\right]=0 .
\end{aligned}
$$

Therefore, Problem (10) can be transformed into the following non-linear PDE problem:

$$
\left\{\begin{aligned}
& H_{t}+k(\theta-m) H_{m}+\frac{\sigma_{m}^{2}}{2} m H_{m m}+\left[\alpha(t)+\lambda_{2} m\right] H_{l}+\frac{\sigma_{L}^{2}}{2} m H_{l l} \\
&+\rho_{S m} \sigma_{m} \sigma_{L} m H_{m l}+\left[(y+l) r-\alpha(t)+m \lambda_{1} \sigma_{L}-\lambda_{2} m\right] H_{y} \\
&-\frac{m}{2 H_{y y}}\left[\sigma_{L}^{2} H_{y l}^{2}+\lambda_{1}^{2} H_{y}^{2}+\sigma_{m}^{2} \rho_{S m}^{2} H_{y m}^{2}+2 \lambda_{1} \sigma_{L} H_{y} H_{y l}\right. \\
&\left.+2 \sigma_{m} \sigma_{L} \rho_{S m} H_{y l} H_{y m}+2 \lambda_{1} \sigma_{m} \rho_{S m} H_{y} H_{y m}\right]=0 \\
& H(T, m, y, l)=(y-u)^{2} .
\end{aligned}\right.
$$

Here we need to point out that it is very difficult to obtain an explicit solution of the complicated non-linear PDE problem for the market is not self-financing (i.e., the manager has a continuous payment $d L(t)$ in the interval $(t, t+d t))$. However, in a particular case, this problem can be solved.

\subsection{Solution of the auxiliary problem (10)}

In this subsection, we shall apply the variable transform techniques and PDE method to a special solution of the auxiliary problem (10). By the terminal condition $H(T, m, y, l)=$ $(y-u)^{2}$, we may look for a candidate solution in the form

$$
H(t, m, y, l)=f(t, m)[y+l-g(t, m, l)]^{2},
$$

where $f(t, m)$ and $g(t, m, l)$ are two undetermined functions with the boundary conditions $f(T, m)=1$ and $g(T, m, l)=l+u$.

After substituting (14) into (13) and by some tedious calculations, we have

$$
\begin{aligned}
{[y+l-g]^{2}\left\{f_{t}+\left[k(\theta-m)-2 \lambda_{1} \sigma_{m} \rho_{S m} m\right] f_{m}+\frac{\sigma_{m}^{2}}{2} m f_{m m}\right.} \\
\left.\quad-m \sigma_{m}^{2} \rho_{S m}^{2} \frac{f_{m}^{2}}{f}+\left[2 r-m \lambda_{1}^{2}\right] f\right\}-2 f[y+l-g]\left\{g_{t}+[k(\theta-m)\right. \\
\left.\quad-m \sigma_{m}^{2}\left(\rho_{S m}^{2}-1\right) \frac{f_{m}}{f}-m \lambda_{1} \sigma_{m} \rho_{S m}\right] g_{m}+\frac{\sigma_{m}^{2}}{2} m g_{m m}+\frac{\sigma_{L}^{2}}{2} m g_{l l}+[\alpha(t) \\
\left.\left.\quad+\lambda_{2} m-m \sigma_{L} \lambda_{1}\right] g_{l}+m \sigma_{L} \sigma_{m} \rho_{S m} g_{l m}-r g\right\}+m f g_{m}^{2} \sigma_{m}^{2}\left(1-\rho_{S m}^{2}\right)=0,
\end{aligned}
$$

where $f$ and $g$ are short for $f(t, m)$ and $g(t, m, l)$, respectively.

Note that Eq. (15) can be taken as a polynomial of variable $y+l-g$. Thus, by the boundary conditions $f(T, m)=1$ and $g(T, m, l)=l+u$, we have

$$
\left\{\begin{array}{l}
f_{t}+\left[k(\theta-m)-2 \lambda_{1} \sigma_{m} \rho_{S m} m\right] f_{m}+\frac{\sigma_{m}^{2}}{2} m f_{m m} \\
\quad-m \sigma_{m}^{2} \rho_{S m}^{2} \frac{f_{m}^{2}}{f}+\left[2 r-m \lambda_{1}^{2}\right] f=0, \\
f(T, m)=1
\end{array}\right.
$$




$$
\begin{aligned}
& \left\{\begin{array}{l}
g_{t}+\left[k(\theta-m)-m \sigma_{m}^{2}\left(\rho_{S m}^{2}-1\right) \frac{f_{m}}{f}-m \lambda_{1} \sigma_{m} \rho_{S m}\right] g_{m}+\frac{\sigma_{m}^{2}}{2} m g_{m m} \\
\quad+\frac{\sigma_{L}^{2}}{2} m g_{l l}+\left[\alpha(t)-m \sigma_{L} \lambda_{1}+m \lambda_{2}\right] g_{l}+m \sigma_{L} \sigma_{m} \rho_{S m} g_{l m}-r g=0 \\
g(T, m, l)=l+u,
\end{array}\right. \\
& m f g_{m}^{2} \sigma_{m}^{2}\left(1-\rho_{S m}^{2}\right)=0 .
\end{aligned}
$$

In what follows, we aim to solve the two terminal value problems (16) and (17) based on Eq. (18). We first solve the problem (16) and postulate that $f(t, m)$ has the following exponential affine form:

$$
f(t, m)=\mathrm{e}^{A_{1}(t) m+A_{2}(t)}
$$

where $A_{1}(t)$ and $A_{2}(t)$ are two undetermined functions with the boundary conditions $A_{1}(T)=0$ and $A_{2}(T)=0$.

Note that $\sigma_{m}>0, m>0$ and $f>0$. Then Eq. (18) is equivalent to $g_{m}=0$ (false) or $\rho_{S m}^{2}=1$. In such a case, substituting (19) into (16) and by some simplifications, Problem (16) can be decomposed into the following ordinary differential equation (ODE) problems:

$$
\begin{aligned}
& \left\{\begin{array}{l}
\frac{d A_{1}(t)}{d t}=\frac{\sigma_{m}^{2}}{2} A_{1}^{2}(t)+\left(k+2 \lambda_{1} \sigma_{m} \rho_{S m}\right) A_{1}(t)+\lambda_{1}^{2}, \\
A_{1}(T)=0,
\end{array}\right. \\
& \left\{\begin{array}{l}
\frac{d A_{2}(t)}{d t}+k \theta A_{1}(t)+2 r=0, \\
A_{2}(T)=0 .
\end{array}\right.
\end{aligned}
$$

The following proposition presents an explicit solution for Problem (20).

Proposition 1 The solution to Problem (20) is given by

$$
A_{1}(t)= \begin{cases}\frac{n_{1} n_{2}\left(1-\mathrm{e}^{\sqrt{\Delta}(T-t)}\right)}{n_{1}-n_{2} \mathrm{e}^{\sqrt{\Delta}(T-t)}}, & \rho_{S m}^{2}=1 \text { and } \Delta>0 \\ \frac{\sigma_{m}^{2}(T-t) n^{2}}{\sigma_{m}^{2}(T-t) n-2}, & \rho_{S m}^{2}=1 \text { and } \Delta=0 \\ \sqrt{\frac{-\Delta}{\sigma_{m}^{4}}} \tan \left(\arctan \left(\frac{k+2 \lambda_{1} \sigma_{m} \rho_{S m}}{\sqrt{-\Delta}}\right)-\frac{\sqrt{-\Delta}(T-t)}{2}\right)+n, & \rho_{S m}^{2}=1 \text { and } \Delta<0\end{cases}
$$

where

$$
\begin{aligned}
& \Delta=\left(k+2 \lambda_{1} \sigma_{m} \rho_{S m}\right)^{2}-2 \lambda_{1}^{2} \sigma_{m}^{2}, \\
& n_{1}=\frac{-\left(k+2 \lambda_{1} \sigma_{m} \rho_{S m}\right)+\sqrt{\Delta}}{\sigma_{m}^{2}}, \\
& n_{2}=\frac{-\left(k+2 \lambda_{1} \sigma_{m} \rho_{S m}\right)-\sqrt{\Delta}}{\sigma_{m}^{2}}, \\
& n=\frac{-\left(k+2 \lambda_{1} \sigma_{m} \rho_{S m}\right)}{\sigma_{m}^{2}} .
\end{aligned}
$$

Proof See the Appendix. 
According to the result of Proposition 1, the solution to Problem (21) can be expressed in terms of $A_{1}(t)$, that is,

$$
A_{2}(t)=\int_{t}^{T} k \theta A_{1}(s) d s+2 r(T-t)
$$

Furthermore, we have the following proposition.

Proposition 2 The solution to Problem (16) is given by

$$
f(t, m)=\mathrm{e}^{A_{1}(t) m+A_{2}(t)}
$$

where $A_{1}(t)$ and $A_{2}(t)$ are given by Eqs. (22) and (23), respectively.

We now solve Problem (17). Let

$$
g(t, m, l)=A_{3}(t) l+A_{4}(t) m+A_{5}(t)
$$

where $A_{3}(t), A_{4}(t)$ and $A_{5}(t)$ are three undetermined functions with the boundary conditions $A_{3}(T)=1, A_{4}(T)=0$ and $A_{5}(T)=u$.

Substituting (24) into (17) and by some simplifications, Problem (17) can be decomposed into the following ODE problems:

$$
\begin{aligned}
& \left\{\begin{array}{l}
\frac{d A_{3}(t)}{d t}-r A_{3}(t)=0, \\
A_{3}(T)=1,
\end{array}\right. \\
& \left\{\begin{array}{l}
\frac{d A_{4}(t)}{d t}-\left(k+\lambda_{1} \sigma_{m} \rho_{S m}+r\right) A_{4}(t)-\left(\lambda_{1} \sigma_{L}-\lambda_{2}\right) A_{3}(t)=0, \\
A_{4}(T)=0,
\end{array}\right. \\
& \left\{\begin{array}{l}
\frac{d A_{5}(t)}{d t}-r A_{5}(t)+k \theta A_{4}(t)+\alpha(t) A_{3}(t)=0, \\
A_{5}(T)=u
\end{array}\right.
\end{aligned}
$$

By a simple calculation, we have

$$
A_{3}(t)=\mathrm{e}^{r(t-T)} .
$$

Then the solutions of Problems (26)-(27) are given by

$$
A_{4}(t)= \begin{cases}-\left(\lambda_{1} \sigma_{L}-\lambda_{2}\right) \mathrm{e}^{r(t-T)}(T-t), & k+\lambda_{1} \sigma_{m} \rho_{S m}=0, \\ \frac{\left.\left(\lambda_{1} \sigma_{L}-\lambda_{2}\right) e^{r(t-T)} \mathrm{e}^{\left(k+\lambda_{1} \sigma_{m} \rho_{S m}\right)(t-T)}-1\right]}{k+\lambda_{1} \sigma_{m} \rho_{S m}}, & k+\lambda_{1} \sigma_{m} \rho_{S m} \neq 0,\end{cases}
$$

and

$$
A_{5}(t)=\left\{u+\int_{t}^{T}\left[k \theta A_{4}(s)+\alpha(s) A_{3}(s)\right] \mathrm{e}^{r(T-s)} d s\right\} \mathrm{e}^{r(t-T)},
$$

respectively. Furthermore, we have the following conclusion. 
Proposition 3 The solution to Problem (17) is given by

$$
g(t, m, l)=A_{3}(t) l+A_{4}(t) m+A_{5}(t)
$$

where $A_{3}(t), A_{4}(t)$ and $A_{5}(t)$ are given by Eqs. (28), (29) and (30), respectively.

Based on the results of Propositions 2-3 and the expression for $H(t, m, y, l)$ in Eq. (14), we have

$$
H(t, m, y, l)=\mathrm{e}^{A_{1}(t) m+A_{2}(t)}\left[y-A_{4}(t) m+\left(1-A_{3}(t)\right) l-A_{5}(t)\right]^{2} .
$$

Obviously, $H_{y y}=2 \mathrm{e}^{A_{1}(t) m+A_{2}(t)}>0$, which means that Problem (10) does have the optimal solution. Substituting (31) into (12) further reveals the optimal investment strategy of the optimal control problem (10) as

$$
\pi^{*}(t)=\frac{\sigma_{L} A_{3}(t)+\sigma_{m} \rho_{S m} A_{4}(t)-\lambda_{1}\left[X(t)-A_{3}(t) l-A_{4}(t) m-A_{5}(t)\right]}{X(t)} .
$$

To sum up, we have the following theorem.

Theorem 1 For any $t \in[0, T]$, the value function of the optimization problem (10) under the condition of $\rho_{S m}^{2}=1$ is given by Eq. (31), while the corresponding optimal solution is given by Eq. (32).

\subsection{Efficient investment strategy and efficient frontier}

In this subsection, we shall apply the Lagrange duality theorem (see Luenberger [19]) to derive the efficient investment strategy of Problem (6). Since the optimal control problem (9) is the same as the optimal control problem (10) when $t=0$, the value function of Problem (9) is

$$
H\left(0, m_{0}, y_{0}, l_{0}\right)=\mathrm{e}^{A_{1}(0) m_{0}+A_{2}(0)}\left[y_{0}-A_{4}(0) m_{0}+\left(1-A_{3}(0)\right) l_{0}-A_{5}(0)\right]^{2}
$$

where $y_{0}=Y(0)$ and $l_{0}=L(0)$. By the analysis of Sect. 3.1, to solve the original M-V portfolio selection problem, we only need to maximize the following function:

$$
\begin{aligned}
J(Y(0), 0 ; \pi(t), \gamma)= & \mathrm{e}^{A_{1}(0) m_{0}+A_{2}(0)}\left[y_{0}-A_{4}(0) m_{0}\right. \\
& \left.+\left(1-A_{3}(0)\right) l_{0}-A_{5}(0)\right]^{2}-\gamma^{2}
\end{aligned}
$$

over $\gamma$. By the expression of $A_{5}(t)$ and $u=C-\gamma$, Eq. (34) can be reduced to

$$
\begin{aligned}
J(Y(0), 0 ; \pi(t), \gamma)= & \gamma^{2}\left[\mathrm{e}^{A_{1}(0) m_{0}+A_{2}(0)-2 r T}-1\right]-2 \gamma \mathrm{e}^{A_{1}(0) m_{0}+A_{2}(0)-2 r T}\{C \\
& -\mathrm{e}^{r T}\left[y_{0}-A_{4}(0) m_{0}+\left(1-A_{3}(0)\right) l_{0}\right. \\
& \left.\left.-\int_{0}^{T}\left(k \theta A_{4}(s)+\alpha(s) A_{3}(s)\right) \mathrm{e}^{-r s} d s\right]\right\}
\end{aligned}
$$




$$
\begin{aligned}
& +\mathrm{e}^{A_{1}(0) m_{0}+A_{2}(0)-2 r T} C^{2} \\
& +\mathrm{e}^{A_{1}(0) m_{0}+A_{2}(0)}\left[y_{0}-A_{4}(0) m_{0}+\left(1-A_{3}(0)\right) l_{0}\right. \\
& \left.-\int_{0}^{T}\left(k \theta A_{4}(s)+\alpha(s) A_{3}(s)\right) \mathrm{e}^{-r s} d s\right]^{2} \\
& -2 C \mathrm{e}^{A_{1}(0) m_{0}+A_{2}(0)-r T}\left[y_{0}-A_{4}(0) m_{0}+\left(1-A_{3}(0)\right) l_{0}\right. \\
& \left.-\int_{0}^{T}\left(k \theta A_{4}(s)+\alpha(s) A_{3}(s)\right) \mathrm{e}^{-r s} d s\right] .
\end{aligned}
$$

Note that Eq. (35) is a quadratic function with respect to $\gamma$, which implies that for (35) there may exist a finite maximum value while the existence of finite maximum value depends on the coefficient of $\gamma^{2}$. For this purpose, in this paper, we need to add one more assumption as follows.

Assumption 2 There exists a constant $m_{0}$ such that $\mathrm{e}^{A_{1}(0) m_{0}+A_{2}(0)-2 r T}-1<0$.

Under Assumption 2, the optimal value of $J(Y(0), 0 ; \pi(t), \gamma)$ can be achieved when $\gamma$ is given by

$$
\gamma^{*}=\frac{C-\left[y_{0}-A_{4}(0) m_{0}+\left(1-A_{3}(0)\right) l_{0}-\int_{0}^{T}\left(k \theta A_{4}(s)+\alpha(s) A_{3}(s)\right) \mathrm{e}^{-r s} d s\right] \mathrm{e}^{r T}}{1-\mathrm{e}^{-\left[A_{1}(0) m_{0}+A_{2}(0)-2 r T\right]}} .
$$

Substituting (36) into (35), the optimal investment strategy and the minimum variance of the M-V ALM problem (9) are given by

$$
\begin{aligned}
\pi^{*}(t)= & \frac{1}{X(t)}\left\{\sigma_{L} A_{3}(t)+\sigma_{m} \rho_{S m} A_{4}(t)-\lambda_{1}\left[X(t)-A_{3}(t) l-A_{4}(t) m\right.\right. \\
& \left.\left.-\left\{C-\gamma^{*}+\int_{t}^{T}\left(k \theta A_{4}(s)+\alpha(s) A_{3}(s)\right) \mathrm{e}^{r(T-s)} d s\right\} \mathrm{e}^{r(t-T)}\right]\right\}
\end{aligned}
$$

and

$$
\begin{aligned}
\operatorname{Var}^{*}[Y(T)]= & \frac{1}{\mathrm{e}^{-\left[A_{1}(0) m_{0}+A_{2}(0)\right]}-\mathrm{e}^{-2 r T}}\left\{C \mathrm{e}^{-r T}-\left[y_{0}-A_{4}(0) m_{0}\right.\right. \\
& \left.+\left(1-A_{3}(0)\right) l_{0}-\int_{0}^{T}\left(k \theta A_{4}(s)+\alpha(s) A_{3}(s)\right) \mathrm{e}^{-r s} d s\right\}^{2},
\end{aligned}
$$

respectively. Furthermore, setting

$$
C^{*}=\mathrm{e}^{r T}\left[y_{0}-A_{4}(0) m_{0}+\left(1-A_{3}(0)\right) l_{0}-\int_{0}^{T}\left(k \theta A_{4}(s)+\alpha(s) A_{3}(s)\right) \mathrm{e}^{-r s} d s\right],
$$

then we can get the global minimum variance $\operatorname{Var}_{\text {min }}^{*}[Y(T)]=0$. Moreover, according to the definition of efficient investment strategy, the rational investors should not select the expected terminal wealth less than $C^{*}$.

In conclusion, we summarize the above results in the following theorem. 
Theorem 2 Under Assumptions 1-2, the efficient investment strategy and the efficient frontier of this $M-V A L M$ problem under the condition of $\rho_{S m}^{2}=1$ for $C \geq C^{*}$ are given by Eqs. (37) and (38), respectively.

Remark 2 Based on the result of Theorem 2, we can derive the efficient investment strategy and efficient frontier for the corresponding asset allocation problem (i.e., the case of no liability).

\section{Numerical analysis}

In this section, we give some numerical examples to analyze the sensitivity of the efficient frontier with respect to the parameters derived from the Heston model. The basic parameters in the model are given by

$$
\begin{aligned}
& r=0.05, \quad \lambda_{1}=-4, \quad k=5, \quad \theta=0.0169, \quad \sigma_{m}=0.25, \quad m_{0}=0.0225, \\
& \alpha(t)=0.05, \quad \lambda_{2}=0.5, \quad \sigma_{L}=2, \quad X_{0}=1, \quad L_{0}=0.1, \quad \rho_{S m}=1, \\
& t=0, \quad T=5 .
\end{aligned}
$$

Here the above parameters are the same as those in Li et al. [18] and satisfy Assumptions $1-2$. We now analyze how the main parameters in the model affect the efficient frontier.

From Fig. 1 , we can see clearly that, as the parameter $\lambda_{1}$ increases from -5 to 5 , the efficient frontier rapidly moves upwards. This means that the expected terminal wealth with the same terminal variance $\operatorname{Var}[Y(T)]$ becomes higher. The main reason is that by Eq. (2), the parameter $\lambda_{1}$ determines the additional appreciation rate of the stock. In the case of other parameters unchanged, the bigger $\lambda_{1}$ leads to the higher additional appreciation rate of the stock, which makes the manager get the higher expected returns by investing in the stock.

Figures 2-4 depict the impacts of the parameters $k, \sigma_{m}, m_{0}$ in the Heston model on the efficient frontiers. From Fig. 2, we find that, as the mean-reversion rate $k$ increases, the efficient frontier moves downwards, i.e., the terminal variance $\operatorname{Var}[Y(T)]$ with the same expected terminal wealth $\mathrm{E}[Y(T)]$ becomes larger. This is mainly because in the case of other parameters having remained unchanged, a bigger $k$ means a higher risk from stochastic volatility, which makes the terminal variance larger. In Fig. 3, we find that when $\sigma_{m}$ increases from 0.0225 to 0.0625 , the efficient frontier moves up and the expected terminal wealth increases rapidly. In this case, the manger adopts a more aggressive investment

Figure 1 Impact of $\lambda_{1}$ on the efficient frontier

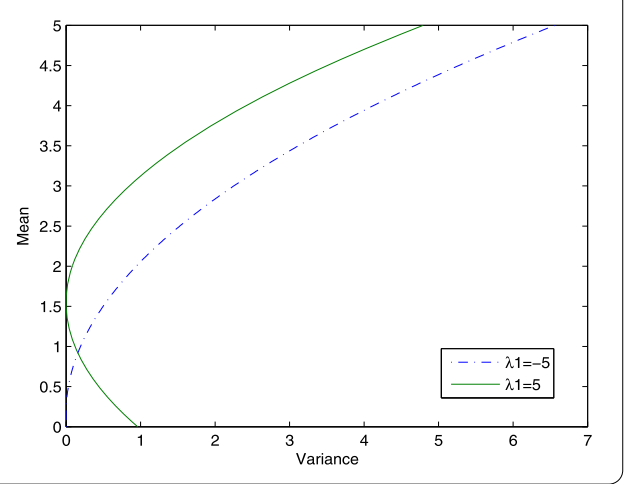


Figure 2 Impact of $k$ on the efficient frontier

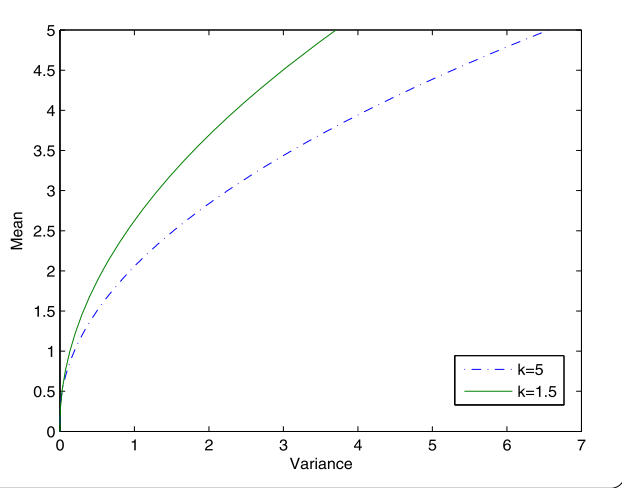

Figure 3 Impact of $\sigma_{m}$ on the efficient frontier

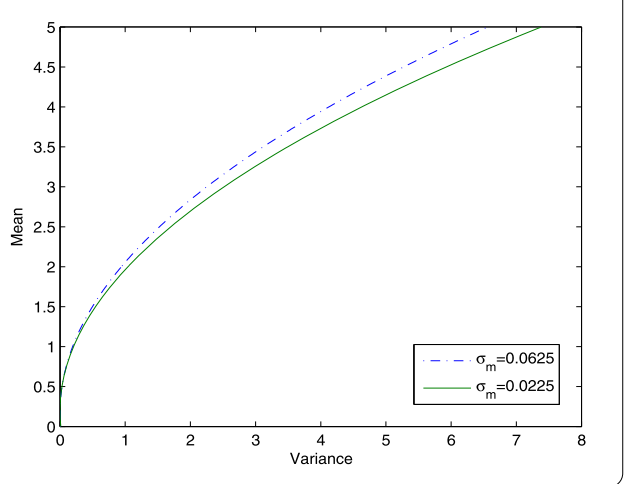

Figure 4 Impact of $m_{0}$ on the efficient frontier

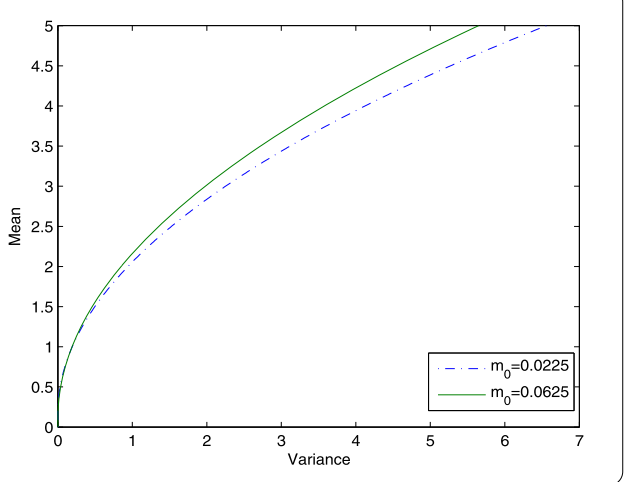

strategy (i.e., more money is allocated to the stock). Similarly, we can analyze the effect of $m_{0}$ on the efficient frontier.

By Eq. (2), we know that the uncertainty of the stock's price is related to the value of $\rho_{S m}$, i.e., if $\rho_{S m}>0$, the uncertainty of the stock's price and its volatility $m(t)$ changes in the same way, while $\rho_{S m}<0$, the uncertainty of $m(t)$ and $S(t)$ changes in the different way. In Fig. 5, we find that when $\rho_{S m}$ varies from -1 to 1 , the efficient frontier moves upward and the expectation improvement increases remarkably. In the current market environment, the manager expects to earn more money from investing in the stock.

In Fig. 6, we can see that the efficient frontier moves down with the increase of $\lambda_{2}$, but at a slow pace. This can be attributed to the fact that in the case of other parameters unchanged, as the parameter $\lambda_{2}$ increases, the appreciation rate of liability becomes higher 
Figure 5 Impact of $\rho_{S m}$ on the efficient frontier

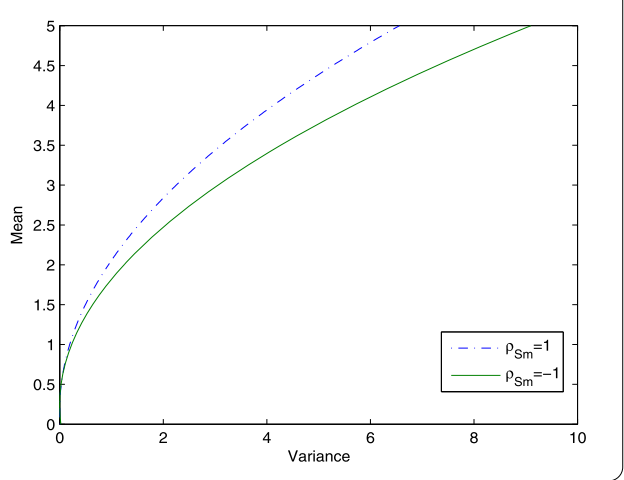

Figure 6 Impact of $\lambda_{2}$ on the efficient frontier

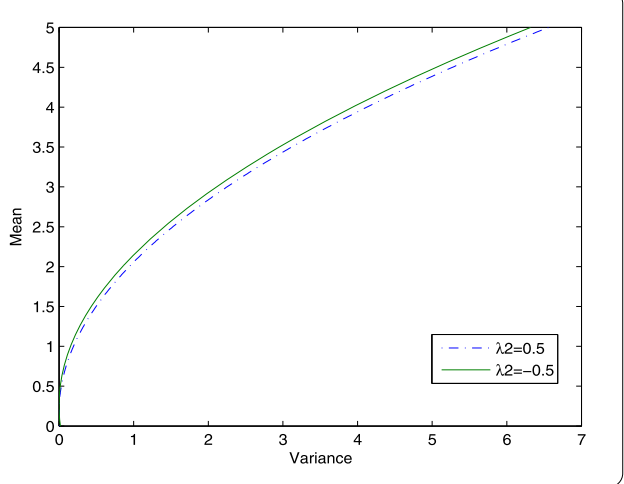

and so is the liability value. To hedge against the risk from the liability, the manager invests more money in the stock, which makes the terminal variance larger.

\section{Conclusions}

In this paper, we study the optimal dynamic M-V ALM problem under the Heston model. Due to some technical difficulties, we only derive the closed-form expressions of the efficient investment strategy and the efficient frontier for the special case: $d W_{S}(t)$ and $d W_{m}(t)$ are perfectly correlated or anti-correlated $\left(\rho_{S m}= \pm 1\right)$. Nevertheless, we are convinced that the results can provides some effective methods for managers in characterizing their optimal portfolio strategies under stochastic volatility environment. Moreover, we provide numerical examples to illustrate how the main parameters of the model affect the efficient frontier. From numerical examples, we find that the effective frontier is obviously affected by the parameters of the stochastic volatility model, which also shows the effectiveness of our model.

\section{Appendix}

In this Appendix, we give the proof of Proposition 1.

Let $\Delta=k^{2}+4 k \lambda_{1} \sigma_{m} \rho_{S m}+2 \lambda_{1}^{2} \sigma_{m}^{2}$ be the discriminant of the following equation:

$$
\frac{1}{2} \sigma_{m}^{2} A_{1}^{2}(t)+\left(k+2 \lambda_{1} \sigma_{m} \rho_{S m}\right) A_{1}(t)+\lambda_{1}^{2}=0 .
$$


If $\Delta>0$, then (39) has two different real roots, namely,

$$
n_{1}=\frac{-\left(k+2 \lambda_{1} \sigma_{m} \rho_{S m}\right)+\sqrt{\Delta}}{\sigma_{m}^{2}}, \quad n_{2}=\frac{-\left(k+2 \lambda_{1} \sigma_{m} \rho_{S m}\right)-\sqrt{\Delta}}{\sigma_{m}^{2}} .
$$

In this case, Problem (20) is equivalent to

$$
\left\{\begin{array}{l}
\frac{d A_{1}(t)}{d t}=\frac{\sigma_{m}^{2}}{2}\left[A_{1}(t)-n_{1}\right]\left[A_{1}(t)-n_{2}\right] \\
A_{1}(T)=0
\end{array}\right.
$$

By applying the separation variable method to the ODE in Problem (40), we have

$$
\frac{d A_{1}(t)}{\left[A_{1}(t)-n_{1}\right]\left[A_{1}(t)-n_{2}\right]}=\frac{\sigma_{m}^{2}}{2} d t .
$$

Furthermore, (41) can be rewritten as

$$
\frac{d A_{1}(t)}{A_{1}(t)-n_{1}}-\frac{d A_{1}(t)}{A_{1}(t)-n_{2}}=\sqrt{\Delta} d t
$$

Integrating (42) on both sides with respect to $t$, we obtain

$$
\int_{t}^{T} \frac{d A_{1}(s)}{A_{1}(s)-n_{1}}-\int_{t}^{T} \frac{d A_{1}(s)}{A_{1}(s)-n_{2}}=\sqrt{\Delta}(T-t)
$$

Combined with the boundary condition $A_{1}(T)=0$, the solution to Problem (40) is

$$
A_{1}(t)=\frac{n_{1} n_{2}\left(1-\mathrm{e}^{\sqrt{\Delta}(T-t)}\right)}{n_{1}-n_{2} \mathrm{e}^{\sqrt{\Delta}(T-t)}} .
$$

If $\Delta=0$, then (39) has only one real root, namely,

$$
n=\frac{-\left(k+2 \lambda_{1} \sigma_{m} \rho_{S m}\right)}{\sigma_{m}^{2}} .
$$

Similar to the former case, $A_{1}(t)$ can be derived by solving the following ODE problem:

$$
\left\{\begin{array}{l}
\frac{d A_{1}(t)}{\left[A_{1}(t)-n\right]^{2}}=\frac{\sigma_{m}^{2}}{2} d t \\
A_{1}(T)=0 .
\end{array}\right.
$$

Integrating the ODE in Problem (43) on both sides with respect to $t$, we have

$$
\int_{t}^{T} \frac{d A_{1}(s)}{\left[A_{1}(s)-n\right]^{2}}=\frac{\sigma_{m}^{2}}{2}(T-t)
$$

Furthermore, by the boundary condition $A_{1}(T)=0$, we obtain

$$
A_{1}(t)=\frac{n^{2} \sigma_{m}^{2}(T-t)}{n \sigma_{m}^{2}(T-t)-2}
$$


If $\Delta<0$, (39) has two imaginary roots. However, we consider the optimization problem in real spaces instead of complex spaces. It is gratifying that in this case $A_{1}(t)$ can be derived by solving the following ODE problem:

$$
\left\{\begin{array}{l}
\frac{d A_{1}(t)}{\left[A_{1}(t)+\frac{k+2 \lambda_{1} \sigma_{m} \rho S m}{\sigma_{m}^{2}}\right]^{2}+\frac{-\Delta}{\sigma_{m}^{4}}}=\frac{\sigma_{m}^{2}}{2} d t \\
A_{1}(T)=0 .
\end{array}\right.
$$

Applying the separation variable method to Problem (44) and after the more complex integral calculation, we have

$$
A_{1}(t)=\sqrt{\frac{-\Delta}{\sigma_{m}^{4}}} \tan \left(\arctan \left(\frac{k+2 \lambda_{1} \sigma_{m} \rho_{S m}}{\sqrt{-\Delta}}\right)-\frac{\sqrt{-\Delta}(T-t)}{2}\right)+n .
$$

Thus, the proof is completed.

\section{Acknowledgements}

The authors wish to thank the anonymous referee for helpful comments, which improved this paper essentially.

\section{Funding}

This research is supported by the National Natural Science Foundation of China (Project No. 11501125), the Science and Technology Project of Jiangxi Provincial Education Department (Project No. GJJ170821) and the Natural Science Foundation of Gannan Normal University (Project No. 201605).

\section{Competing interests}

The authors declare that they have no competing interests.

\section{Authors' contributions}

The authors declare that the study is realized in collaboration with the same responsibility. All authors are read and approved the manuscript.

\section{Author details}

${ }^{1}$ Key Laboratory of Jiangxi Province for Numerical Simulation and Emulation Techniques, Ganzhou, China. ${ }^{2}$ College of Mathematics and Computer Science, Gannan Normal University, Ganzhou, China.

\section{Publisher's Note}

Springer Nature remains neutral with regard to jurisdictional claims in published maps and institutional affiliations.

Received: 23 February 2018 Accepted: 15 June 2018 Published online: 27 July 2018

\section{References}

1. Sharpe, W.F., Tint, L.G.: Liabilities—a new approach. J. Portf. Manag. 16(2), 5-10 (1990)

2. Keel, A., Müller, H.: Efficient portfolios in the asset liability context. ASTIN Bull. 25(1), 33-48 (1995). https://doi.org/10.2143/AST.25.1.563252

3. Leippold, M., Trojani, F., Vanini, P.: A geometric approach to multi-period mean variance optimization of assets and liabilities. J. Econ. Dyn. Control 28(6), 1079-1113 (2004)

4. Chiu, C.M., Li, D.: Asset and liability management under a continuous-time mean-variance optimization framework. Insur. Math. Econ. 39(3), 330-355 (2006)

5. Xie, S.X., Li, Z.F., Wang, S.Y.: Continuous-time portfolio selection with liability: mean-variance model and stochastic LQ approach. Insur. Math. Econ. 42(3), 943-953 (2008)

6. Chen, P., Yang, H.L., Yin, G.: Markowitz's mean-variance asset-liability management with regime switching: a continuous-time model. Insur. Math. Econ. 18(1), 456-465 (2008)

7. Chen, P., Yang, H.L.: Markowitz's mean-variance asset-liability management with regime switching: a multi-period model. Appl. Math. Finance 18(1), 29-50 (2011)

8. Chiu, C.M., Wong, H.Y.: Mean-variance asset-liability management: cointegrated assets and insurance liabilities. Eur. J. Oper. Res. 223(3), 785-793 (2012)

9. Chiu, M.C., Wong, H.Y.: Mean-variance principle of managing cointegrated risky assets and random liabilities. Oper. Res. Lett. 41(1), 98-106 (2013)

10. Yao, H.X., Lai, Y.Z., Li, Y.: Continuous-time mean-variance asset-liability management with endogenous liabilities. Insur. Math. Econ. 52(1), 6-17 (2013)

11. Yao, H.X., Zeng, Y., Chen, S.M.: Multi-period mean-variance asset-liability management with uncontrolled cash flow and uncertain time-horizon. Econ. Model. 30(1), 492-500 (2013) 
12. Chiu, C.M., Wong, H.Y: Mean-variance asset-liability management with asset correlation risk and insurance liabilities. Insur. Math. Econ. 59, 300-310 (2014)

13. Pan, J., Xiao, Q.X.: Optimal mean-variance asset-liability management with stochastic interest rates and inflation risks. Math. Methods Oper. Res. 85(3), 491-519 (2017)

14. Cox, J.C., Ross, S.A.: The valuation of options for alternative stochastic processes. J. Financ. Econ. 3(1), 145-166 (1976)

15. Stein, E.M., Stein, J.C.: Stock price distributions with stochastic volatility: an analytic approach. Rev. Financ. Stud. 4(4), 725-752 (1991)

16. Heston, S.L.: A closed-form solution for options with stochastic volatility with applications to bond and currency options. Rev. Financ. Stud. 6(2), 327-343 (1993)

17. Zhang, M., Chen, P.: Mean-variance asset-liability management under constant elasticity of variance process. Insur. Math. Econ. 70, 11-18 (2016)

18. Li, D.P., Shen, Y., Zeng, Y.: Dynamic derivative-based investment strategy for mean-variance asset-liability management with stochastic volatility. Insur. Math. Econ. 78, 72-86 (2018)

19. Luenberger, D.G.: Optimization by Vector Space Methods. Wiley, New York (1968)

\section{Submit your manuscript to a SpringerOpen ${ }^{\circ}$} journal and benefit from:

- Convenient online submission

- Rigorous peer review

- Open access: articles freely available online

- High visibility within the field

- Retaining the copyright to your article

Submit your next manuscript at $\gg$ springeropen.com 